\title{
Chronic perivascular inoculation with Chlamydophila pneumoniae results in plaque formation in vivo
}

\author{
Markus Georg Engelmann ${ }^{1}$, Constanze Veronika Redl ${ }^{1}$, Jaroslav Pelisek ${ }^{1}$, Corinna Barz ${ }^{2}$, \\ Juergen Heesemann ${ }^{2}$ and Sigrid Nikol ${ }^{1,3}$ \\ ${ }^{1}$ Medical Department I - Cardiology, Ludwig Maximilian University, Munich, Germany; ${ }^{2}$ Max von Pettenkofer \\ Institute of Medical Microbiology, Ludwig Maximilian University, Munich, Germany and ${ }^{3}$ Department of \\ Cardiology and Angiology, University of Muenster, Muenster, Germany
}

\begin{abstract}
Hypercholesterolemic and normocholesterolemic rabbit models of chronic arterial Chlamydophila (Chlamydia) pneumoniae (CPN) inoculation were established and the role of both viable and inactivated bacteria was investigated in atherogenesis. A total of 29 rabbits were randomized to four groups. Groups A and B were fed a cholesterol-enriched diet, and groups C and D were fed a normal diet. Arterial segments of group A and C animals were inoculated in vivo using viable CPN chronically using repeated perivascular applications. Contralateral arteries were treated using heat-inactivated CPN. Group B and D animals were treated with repeated perivascular injections of bacterial lipopolysaccharide (LPS) and saline (control). Additional hypercholesterolemic rabbits were treated by repeated injections using viable and inactivated CPN, each controlled by saline injections. To compare the effects of this chronic inoculation model, additional animals received single injections of either viable CPN, inactivated CPN, LPS, or saline. Vascular tissues ( $n=162$ treated arteries of 29 rabbits) were analyzed using morphometry at histology. CPN was detected by fluorescenceimmunohistochemistry and nested polymerase chain reaction. Only in hypercholesterolemic, but not in normocholesterolemic rabbits, chronic perivascular infection of all bacterial components, viable and heatinactivated CPN, as well as LPS resulted in a significant increase in atheromatous lesion formation (lesion area index: $0.23 \pm 0.08,0.25 \pm 0.09$, and $0.15 \pm 0.05$ ) when compared to controls (lesion area index $0.01 \pm 0.01$, $P=0.002)$. CPN persisted in atheromatous lesions and vascular tissues. Single perivascular infection using CPN or inactivated CPN was not able to induce lesion formation (lesion area index: $0.03 \pm 0.03,0.03 \pm 0.02$ vs $0.03 \pm 0.02$ after single saline inoculation, $P=0.965$ ). In conclusion, chronic vascular infection with CPN or CPN components acts as a cofactor requiring other major atherogenic stimuli, rather than as a causative agent. Laboratory Investigation (2006) 86, 467-476. doi:10.1038/labinvest.3700411; published online 20 March 2006
\end{abstract}

Keywords: atherosclerosis; Chlamydophila pneumoniae; Chlamydia pneumoniae; vascular inflammation; lipopolysaccharide

Chlamydophila (Chlamydia) pneumoniae (CPN) is a human pathogen distributed worldwide, causing acute respiratory diseases such as pneumonia, bronchitis, pharyngitis, and sinusitis. Recently, a possible association of CPN with coronary artery disease and other forms of vascular atherosclerosis was suspected, following seroepidemiological studies and the demonstration of CPN in atherosclerotic plaques obtained in vivo. ${ }^{1-4}$ However, the

Correspondence: Dr MG Engelmann, MD, Medical Department I Cardiology, Ludwig Maximilian University, Klinikum Grosshadern, Marchioninistrasse 15, Munich D-81377, Germany.

E-mail: markus.engelmann@med.uni-muenchen.de

Received 15 December 2005; revised 13 February 2006; accepted 13 February 2006; published online 20 March 2006 pathogenic role of these organisms for the development of atherosclerotic vascular disease and the specificity of chlamydial effects is still poorly understood. In addition, the causal relationship between Chlamydia infection and atherogenesis has not yet been thoroughly investigated.

Several animal models of CPN infections were published mostly for mice, but also for rabbits and primates, investigating intranasal inoculation or subcutaneous, intravenous, and intracerebral infection routes. ${ }^{5-10}$ Apolipoprotein (Apo) E-deficient knockout mice developed accelerated atherosclerosis if infected by intranasal inoculation. ${ }^{11}$ Administration of macrolide antibiotics inhibited increased intimal thickening following intranasal inoculation in New Zealand White (NZW) rabbits fed with 
cholesterol-enriched diet. ${ }^{12}$ In LDL receptor-deficient mice, the atherogenic effects of CPN were demonstrated to be dependent on serum cholesterol levels and specific to CPN. ${ }^{13}$

Although a favorable action on endothelial function has been shown after short-term treatment with azithromycin,${ }^{14}$ large clinical trials demonstrated no beneficial effect of macrolide treatment. ${ }^{15-17}$

The purpose of the present investigations in a rabbit model was to assess the effect of perivascularly administered viable or inactivated CPN, controlled by the administration of Escherichia coli (E. coli) LPS as a typical component of Gramnegative organisms, on lesion formation and plaque induction. Experiments were performed under conditions of hypercholesterolemia as well as normocholesterolemia to analyze the role of CPN under conditions of low atherogenic burden.

\section{Methods}

\section{Animal Study Protocol}

Investigations were carried out with the permission from federal government, conforming to national and local University guidelines on animal experiments. Figure 1 (and in part Table 1) gives an overview of the experimental study design. Female NZW rabbits $(n=29)$, mean weight $3.8 \pm 0.4 \mathrm{~kg}$, were investigated. Of these, 22 experimental animals were analyzed in a chronic perivascular inoculation model. In all, 12 of them were fed an atherogenic diet supplement with $0.5 \%$ cholesterol for 16 weeks, and 10 animals received chop diet. After 3 weeks, they were randomly assigned to four treatment groups. Groups A and C received a perivascular injection of CPN (strain AR-39; Washington Research Foundation, Seattle, WA, USA) along the right auricular artery, right carotid, and right superficial femoral artery. Along contralateral left arteries, heat-inactivated CPN $\left(1 \mathrm{~h} \text { at } 56^{\circ} \mathrm{C}\right)^{18}$ was injected. Bacteria were chronically administered at weeks 3 , 7 , and 11 with a dosage of $10^{6}$ IFU. Groups B and D received repeated perivascular injections of $E$. coli lipopolysaccharide (LPS) (Strain J5, Rc mutant, $1 \mu \mathrm{g}$; Sigma) along the right auricular artery, right carotid, and right superficial femoral artery. Along the contralateral left arteries, sodium chloride was injected at weeks 3,7 , and 11 (control). To exclude influences of injection of viable organisms onto atheromatous lesion formation of the contralateral sites, additional two hypercholesterolemic animals received repeated applications of viable CPN next to auricular, carotid, and femoral arteries $(n=6$ arteries analyzed), and sodium chloride next to the contralateral arteries ( $n=6$ arteries). Two further hypercholesterolemic animals were identically treated using inactivated CPN ( $n=6$ arteries), and sodium chloride ( $n=6$ arteries). Seven animals fed a cholesterol-enriched diet, and received a single injection of either CPN, inactivated CPN, LPS, or sodium chloride ( $n=42$ arterial segments analyzed).

For injection sites, arteries were chosen that are not predisposed to spontaneous lesion formation because of low shear stress, such as auricular, common carotid, and superficial femoral arteries. The injection next to the auricular artery was controlled macroscopically, and the injections next

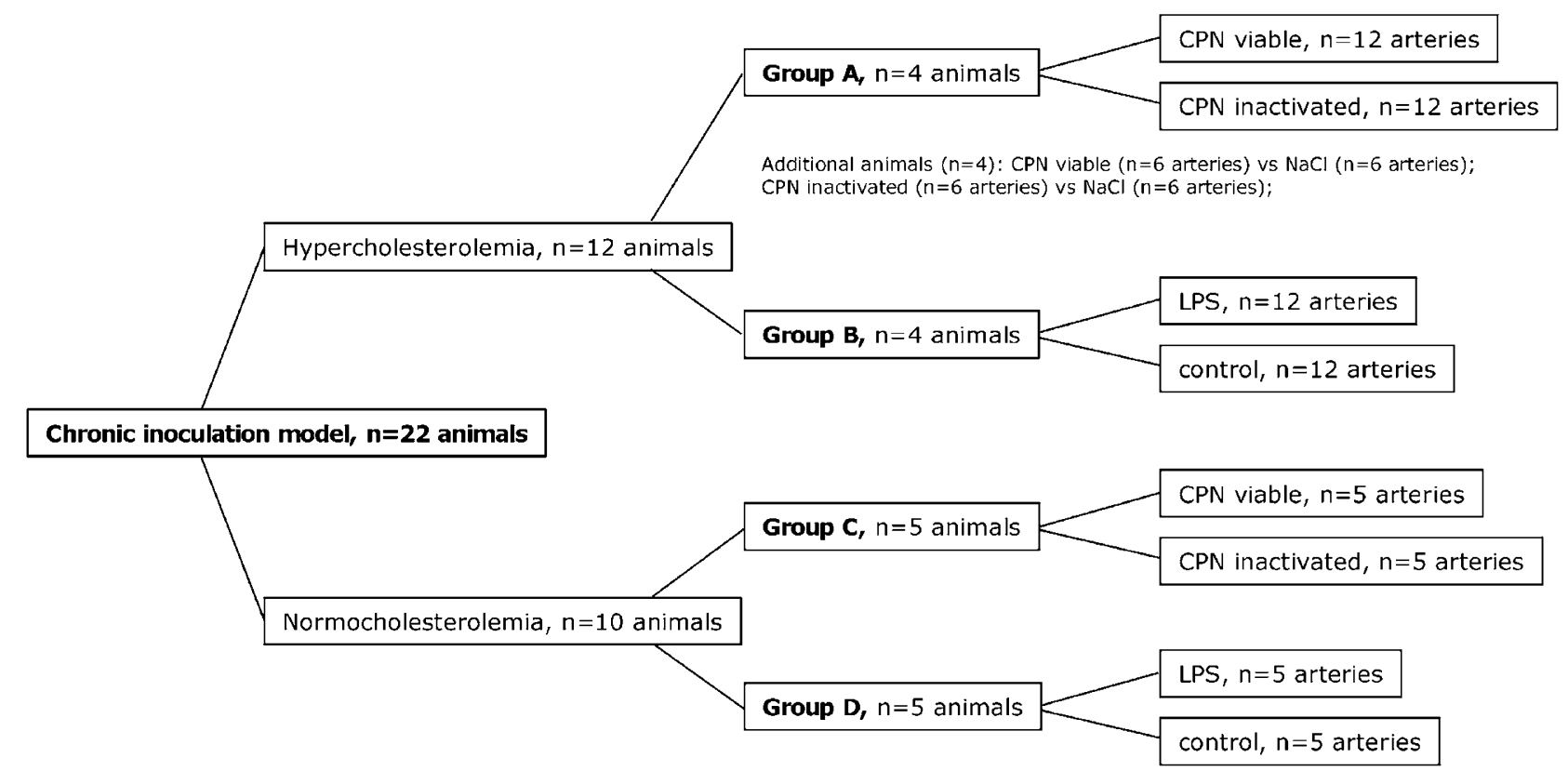

Figure 1 Experimental design of the chronic inoculation model. Animals of the chronic inoculation model were randomized to four groups. Groups A and B were fed a cholesterol-enriched diet, and groups C and D were fed a normal diet. To investigate differences between the chronic and single inoculation, additional seven animals were fed a cholesterol-enriched diet and received bacterial components or saline once after 3 weeks (not shown at the chart). CPN: Chlamydophila pneumoniae, Nacl: saline. 
Table 1 Comparison of atheromatous lesion formation following local perivascular inoculation in rabbit arteries

\begin{tabular}{|c|c|c|c|c|}
\hline Inoculation treatment & No. of rabbits & Intima media ratio & No. of lesions (\%) & Lesion area index \\
\hline \multicolumn{5}{|c|}{ Normocholesterolemia, chronic perivascular inoculation } \\
\hline Saline ( $n=5$ vessel segments) & $l_{n-5}$ & $0.04 \pm 0.01$ & $0 / 0(0 \%)$ & 0.0 \\
\hline LPS $(n=5)$ & $n=5$ & $0.04 \pm 0.01$ & $0 / 0(0 \%)$ & 0.0 \\
\hline CPN viable $(n=5)$ & & $0.03 \pm 0.01$ & $0 / 0(0 \%)$ & 0.0 \\
\hline CPN inactivated $(n=5)$ & $n=5$ & $0.03 \pm 0.01$ & $0 / 0(0 \%)$ & 0.0 \\
\hline \multicolumn{5}{|c|}{ Hypercholesterolemia, chronic perivascular inoculation } \\
\hline Saline ( $n=12$ vessel segments) & & $0.11 \pm 0.01$ & $2 / 12(17 \%)$ & $0.01 \pm 0.01$ \\
\hline LPS $(n=12)$ & $n=4$ & $0.25 \pm 0.05^{*}$ & $10 / 12(83 \%)^{\dagger}$ & $0.15 \pm 0.05^{\ddagger}$ \\
\hline CPN viable $(n=12)$ & & $0.28 \pm 0.08^{*}$ & $9 / 12(75 \%)^{\dagger}$ & $0.23 \pm 0.08^{\ddagger}$ \\
\hline CPN inactivated $(n=12)$ & $n=$ & $0.27 \pm 0.08^{*}$ & $7 / 12(58 \%)^{\dagger}$ & $0.25 \pm 0.09^{\ddagger}$ \\
\hline Saline ( $n=12$ vessel segments) & & $0.11 \pm 0.03$ & $2 / 12(17 \%)$ & $0.02 \pm 0.01$ \\
\hline CPN viable $(n=6)$ & $n=4^{\#}$ & $0.31 \pm 0.06^{* *}$ & $5 / 6(83 \%)^{\dagger \dagger}$ & $0.19 \pm 0.06^{\ddagger \ddagger}$ \\
\hline CPN inactivated $(n=6)$ & & $0.22 \pm 0.07^{* *}$ & $4 / 6(67 \%)^{+\dagger}$ & $0.14 \pm 0.08^{\ddagger \ddagger}$ \\
\hline \multicolumn{5}{|c|}{ Hypercholesterolemia, single perivascular inoculation } \\
\hline Saline ( $n=12$ vessel segments) & & $0.11 \pm 0.02$ & $3 / 12(25 \%)$ & $0.03 \pm 0.02$ \\
\hline LPS $(n=12)$ & $n$ & $0.13 \pm 0.03$ & $3 / 12(25 \%)$ & $0.04 \pm 0.02$ \\
\hline CPN viable $(n=9)$ & & $0.13 \pm 0.02$ & $2 / 9(22 \%)$ & $0.03 \pm 0.03$ \\
\hline CPN inactivated $(n=9)$ & ${ }_{n}$ & $0.11 \pm 0.02$ & $2 / 9(22 \%)$ & $0.03 \pm 0.02$ \\
\hline
\end{tabular}

LPS: lipopolysaccharide; CPN: Chlamydophila pneumoniae.

\#These four additional hypercholesterolemic rabbits were treated by repeated injections using viable and inactivated CPN, each controlled by saline injections on the contralateral vessel. Intima media ratio and lesion area index are expressed in mean \pm s.e.m. Groups were compared using one-way analysis of variance (ANOVA), and $\chi^{2}$ test, respectively:

${ }^{*} P=0.002$ vs control (ANOVA).

${ }^{\dagger} P<0.0001$ vs control $\left(\chi^{2}\right.$ test).

${ }^{\ddagger} P=0.006$ vs control (ANOVA).

${ }^{* *} P=0.023$ vs control (ANOVA).

$\because P=0.014$ vs control ( $\chi^{2}$ test).

${ }^{\ddagger} P=0.017$ vs control (ANOVA).

to the carotid and femoral arteries were guided by an $8 \mathrm{MHz}$ Doppler ultrasonic probe (KMS 5041, Doppler 762; Kranzbühler, Solingen, Germany) using $1 \mathrm{ml}$ syringes and $25 \mathrm{G}$ needles. Near auricular arteries, a volume of $100 \mu \mathrm{l}$ was administered, and other vessels received $200 \mu \mathrm{l}$ total volume. Serum cholesterol and triglycerides were measured using routine techniques at weeks 6 and 16 in all animals.

\section{Histopathological Studies}

Rabbits were killed using intravenous pentobarbital. Carotid, superficial femoral and, auricular arteries, and aortas were removed, preserving surrounding adventitial tissues. After dividing into transversal segments, vessel sections were embedded in OCT (Sakura, Zoetervoude, The Netherlands) and snapfrozen in liquid nitrogen. Sections $(10 \mu \mathrm{m})$ were stained with hematoxylin and eosin, and van Giesson's elastic staining. A total of 162 treated arterial sites were analyzed. Four segments were stained per treated site, and the segment with the highest plaque area was chosen for quantitative analysis. Tissue slides were scanned at $\times 4$ magnification with a microscope linked to a chargecoupled device camera (Nikon 104, Duesseldorf, Germany) and analyzed using computer-assisted morphometry (Adobe Photoshop version 5.0; Adobe
Systems Inc., San Jose, CA, USA). Intima media ratio was calculated from the measured area of intima and area of media, and lesion area index from the lesion area related to the area of media, as published previously. ${ }^{19}$ To prevent bias from an operator-dependent effect, morphometric analyses were performed independently of the operator (MGE) by CVR who was blinded towards the perivascular treatment performed.

CPN organisms were also detected by immunofluorescence using a specific monoclonal antibody, which was labeled by Cy2 (specific antibody to Chlamydia major outer membrane protein; VIVA Diagnostik, Hamburg, Germany). Positive controls were performed using CPN-infected human Hep-2 cells (Bios Laboratory Diagnostics, Graefelfing, Germany). All immunohistochemical analyses were controlled using isotype-matched antibodies, carried out to prove the specificity of staining. A semiquantitative analysis of CPN antigen was performed to determine whether it is present, weak, or absent in the intima, media, or adventitia.

\section{Analysis of Rabbit C-Reactive Protein (CRP) Serum Levels}

To assess the degree of systemic inflammation in rabbits after either LPS or CPN injections, serum 
levels of CRP were analyzed by enzyme-linked immunosorbent assay using purified chicken antirabbit CRP antibody conjugated with horseradish peroxidase and tetramethylbenzidine/hydrogen peroxide as chromogen substrate (Immunology Consultant Laboratory Inc., Newberg, OR, USA). The positive control was adjusted to contain $80 \mathrm{ng} / \mathrm{ml}$ of rabbit CRP. Absorbance of the final reaction was determined at $450 \mathrm{~nm}$. Serum of hypercholesterolemic rabbits treated with repeated applications of LPS (group A animals, $n=4$ ), and animals that received recurrent CPN injections (Group B animals, $n=4$ ) was obtained before perivascular injection (baseline), 5 days after perivascular application (week 4, time point of the maximum local reaction), and before being killed (week 16).

\section{Polymerase Chain Reaction (PCR) Analysis}

Frozen tissue was digested with proteinase K. CPN DNA was detected by a nested PCR. Following primers were used: Cpn104V (GCGGAAGGGTTAG TAGTA), Cpn654R (CCCTTTTCCCCATCTATC), PCR product $570 \mathrm{bp}$; Ctr182V (GATATTTGGGCATCCGC), Ctr625R (TAGTATTAGATGCCGACTC), PCR product $460 \mathrm{bp}$ in 30 cycles each, with an annealing temperature of $60^{\circ} \mathrm{C}$. Molecular weight marker VIII obtained from Boehringer Mannheim (Mannheim, Germany) was used for size calculation. Molecular weight marker VIII is a mixture of pUC21 cleaved with HpaII and, for the lower molecular weight range, pUC21 with HindIII/DraI.

\section{Statistical Analysis}

Results are expressed in means \pm s.e. Intima media ratio and lesion area index between groups were compared by one-factorial analysis of variance. Sample sizes determined to assess a difference of 0.15 in lesion area index with an $80 \%$ power and an $\alpha$ error of $5 \%$ would require six segments for each treatment group. The number of lesions occurring in each group was compared using $\chi^{2}$ test. A level of $P<0.05$ was taken to indicate statistical significance (SPSS release 12.0.1, SPSS Inc., Chicago, IL, USA).

\section{Results}

\section{Clinical and Laboratory Findings}

Perivascular injections of viable or inactivated CPN or LPS resulted in transient development of erythema at the auricular application site. The peak skin reaction was observed at day 1 after application and differed in size significantly from the site of control injection. Mild erythema lasted for 4 days and resolved in all animals. Serum cholesterol at week 16 did not differ between both hypercholesterolemic groups (group A: $1440 \pm$
$320 \mathrm{mg} / \mathrm{dl}$; group B: $1637 \pm 520, P=\mathrm{NS}$ ). Following repeated LPS/saline applications (group A), hypercholesterolemic animals demonstrated a slight increase of CRP levels, which persisted until being killed (CRP at baseline: $0.03 \pm 0.001 \mathrm{mg} / \mathrm{dl}$; week 4: $0.28 \pm 0.13 \mathrm{mg} / \mathrm{dl}$; week $16: 0.28 \pm 0.09 \mathrm{mg} / \mathrm{dl}$, $P=0.15)$. After chronic CPN viable/inactivated applications, hypercholesterolemic rabbits (group B) developed similar CRP elevations (CRP at baseline: $0.04 \pm 0.006 \mathrm{mg} / \mathrm{dl}$; week $4: 0.65 \pm 0.32 \mathrm{mg} / \mathrm{dl}$; week 16: $0.43 \pm 0.1 \mathrm{mg} / \mathrm{dl}, P=0.13$ ). The serum CRP levels did not differ significantly between LPS- and CPN-treated animals (baseline: $P=0.24$; week 4: $P=0.32$; week $16: P=0.31$ ).

\section{Effects of CPN on Lesion Development}

Repeated perivascular application of viable CPN resulted in an increase of atheromatous lesion formation in comparison to control injections using sodium chloride after 16 weeks of high cholesterol feeding. Following repeated infection with viable $\mathrm{CPN}$, in nine out of 12 vessels, atheromatous lesions were detectable compared to two out of 12 control vessels after treatment with saline. Lesion area index following chronic infection with viable CPN was also significantly increased $(0.23 \pm 0.08$ vs $0.01 \pm 0.01, P=0.006$; Figure 2a) Atheromatous lesion induction was also observed in arterial segments treated with either inactivated CPN or $E$. coli LPS (lesion area index was $0.25 \pm 0.09$, $0.15 \pm 0.05$ vs $0.01 \pm 0.01, P=0.006$; Figure 2a, Table 1 ), although plaque formation was more prominent in vessel segments following inoculation using viable CPN. Figure 3 shows representative hematoxylin and eosin stainings of carotid arteries following perivascular inflammation in hypercholesterolemic animals. Lesions consisted mainly of macrophages and smooth muscle cells. Chronic inactivated CPN inoculation also induced an increased lesion formation when compared to controls (seven out of $12 \mathrm{vs}$ two out of $12, P<0.001$; see Figure $2 \mathrm{~b}$, Table 1 ). The histomorphology of the lesions corresponded to stage II of the Stary classification with predominating macrophages and vascular smooth muscle cells (VSMCs). ${ }^{20}$ Following chronic infection, arteries treated with viable CPN demonstrated no or only scarce presence of leukocytes in the adventitia and no infiltration of the media. After repeated injections of inactivated CPN or LPS, no cellular infiltration was observed.

Histomorphological analysis of the four additional hypercholesterolemic animals, which were treated by repeated injections using viable, and inactivated CPN, respectively, each controlled by sodium chloride injections next to the contralateral arteries demonstrated similar findings. Atheromatous lesions were observed in two out of $12(17 \%)$ segments in control vessels compared to five out of six segments (viable CPN, 83\%) and four out of six segments $(67 \%)$ after repeated application of 


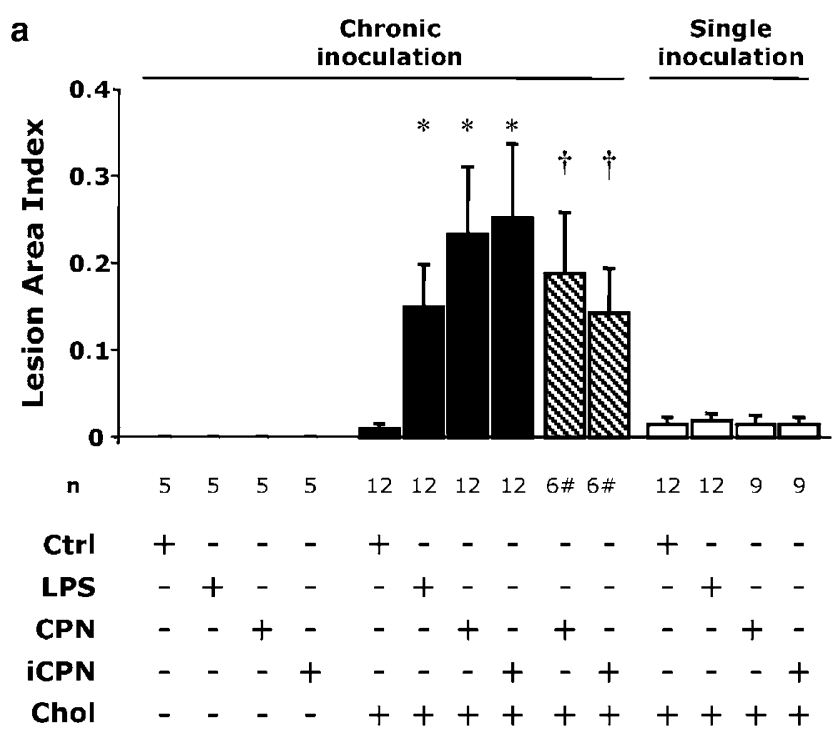

b

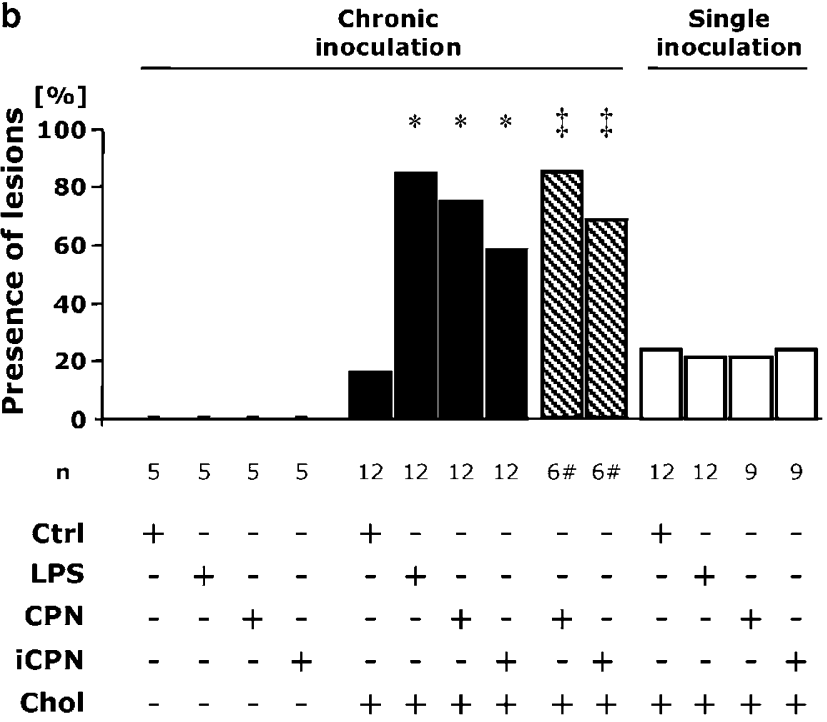

Figure 2 Atheromatous lesion formation following chronic and single bacterial inoculation. Graph shows lesion area index (a) and occurrence of lesions (in \%) (b) in both normocholesterolemic and hypercholesterolemic animals. Ctrl: saline injections; LPS: lipopolysaccharide; CPN: viable Chlamydophila pneumoniae; iCPN: heat-inactivated CPN. Lesion area index in normaldiet groups was 0.0 as no lesions developed. $n=$ number of segments treated; ${ }^{*} P<0.001,{ }^{\dagger} P=0.017,{ }^{\ddagger} P=0.014$ when compared to control. "lesion area index and lesion formation of additional rabbits that received repeated applications of CPN, or iCPN next to arteries, and sodium chloride next to the contralateral arteries to exclude influences of injection of organisms onto atheromatous lesion formation of the contralateral sites.

inactivated CPN $(P=0.014)$. Lesion area index was also significantly increased following viable $(0.19 \pm 0.06)$ or inactivated CPN injections $(0.14 \pm$ 0.08) when compared to saline control segments $(0.02 \pm 0.01, P=0.017)$.

The single inoculation of neither viable CPN, heat-inactivated CPN, nor LPS in hypercholesterolemic rabbits resulted in plaque formation (lesion area index: $0.03 \pm 0.03,0.03 \pm 0.02,0.04 \pm 0.02$ vs $0.03 \pm 0.02$ in saline-treated controls, $P=0.965$; see Table 1, Figure 2a and b).

Under conditions of normocholesterolemia, neither atheromatous lesion formation nor intimal thickening was observed following repeated applications (lesion area index 0.0 vs $0.0, P=1.0$, viable $\mathrm{CPN}$ vs inactivated organisms; Figure $2 \mathrm{a}$ and $\mathrm{b}$ ).

\section{Vascular Presence of CPN after Perivascular Infection}

CPN DNA was detected by nested immunofluorescence and PCR in eight out of 12 arterial segments $(67 \%)$ treated repeatedly with viable CPN (Figures 4 and 5). CPN persisted predominantly in macrophage-rich lesions (Figure 4a), but were also detected in media and in the adventitia to a comparable extent (Figure 4c and d). Six out of 12 segments $(50 \%)$ treated with inactivated CPN were also positive using PCR $(P=0.680)$, whereas CPN antigen detection in these arterial tissues was negative or very weak using immunofluorescence. Arteries treated by repeated inoculation of LPS were tested to be negative for Chlamydia, either by PCR or by immunofluorescence.

\section{Systemic Spread of CPN after Perivascular Infection}

In remote tissue specimens (lung, spleen, lymph nodes), CPN DNA was detected to be very weak in lung tissue and paracarotid lymph nodes by nested PCR in one animal. In all other tissues CPN were absent.

\section{Discussion}

Although infection with CPN has been associated with atherosclerosis, ${ }^{21}$ the role of the organism in the context of atherogenesis is still not sufficiently defined to draw conclusions regarding clinical relevance. $^{22}$ Using hypercholesterolemic rabbits, we have demonstrated that chronic perivascular application of CPN or its components results in increased atheromatous lesion formation. Previous investigations comprised a variety of in vivo models (mostly mice, rabbits) to assess a possible causal relationship between $\mathrm{CPN}$ and atherogenesis. In these models, an intranasal route was used to generate the chlamydial infection. In LDL receptordeficient mice, the infection with CPN AR-39 resulted in significant plaque formation and was shown to be specific for CPN. ${ }^{13}$ The disadvantage of intranasal inoculation models is that the location of manifestation of infected plaque lesions is not predictable owing to the systemic application route. ${ }^{23,24,25}$ The local intramural access by Muhlestein et al and the perivascular approach in the present investigation address this problem. Local inflammations or infections of arterial segments were created by perivascular inoculation in order to investigate 

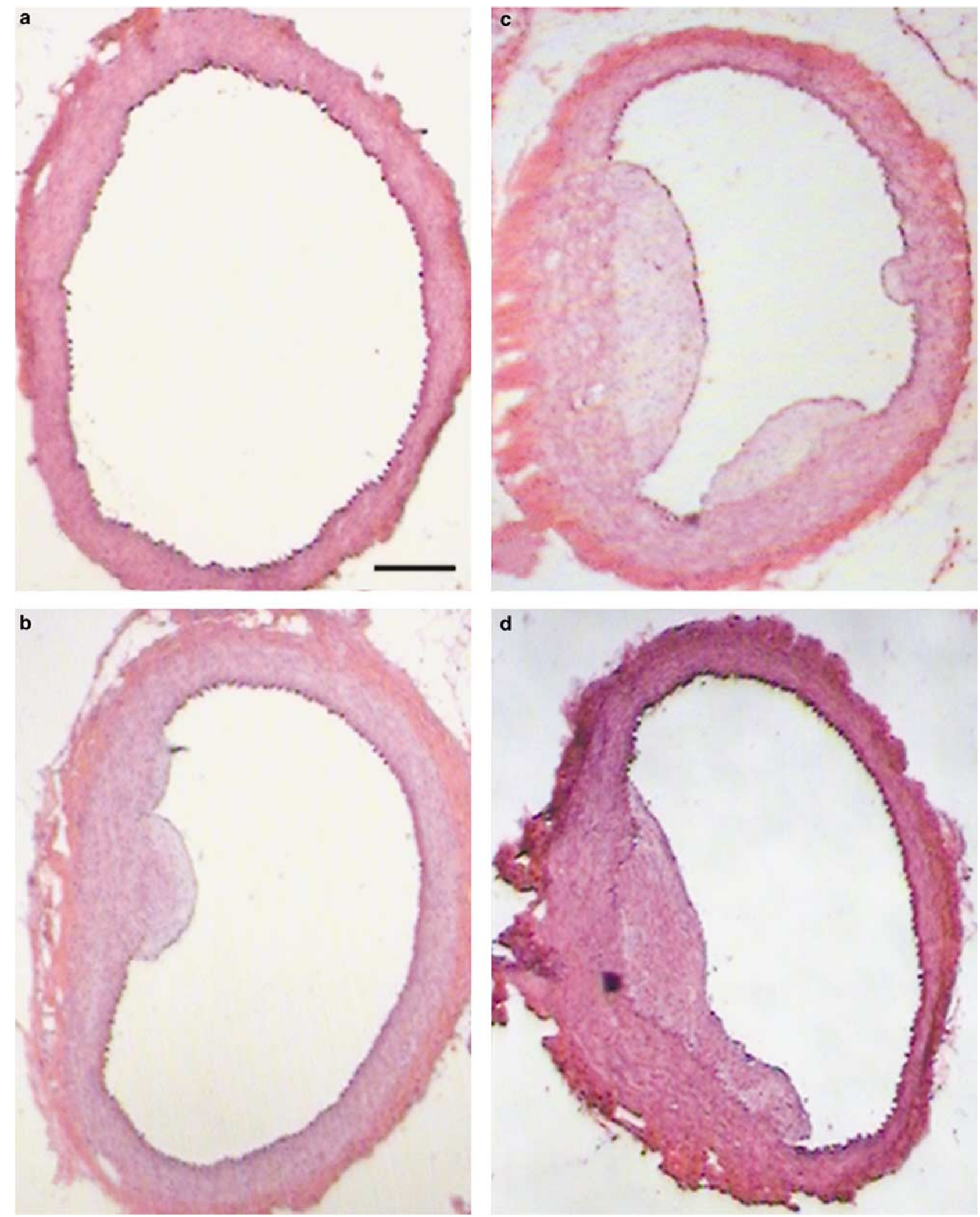

Figure 3 Histomorphology of arterial vessels following chronic perivascular inoculation. Hematoxylin and eosin staining of carotid arteries following chronic perivascular inoculation in hypercholesterolemic experimental animals. (a) saline; (b) lipopolysaccharide; (c) inactivated C. pneumoniae (CPN); and (d) chronic infection using viable CPN. Bar represents $250 \mu \mathrm{m}$. 

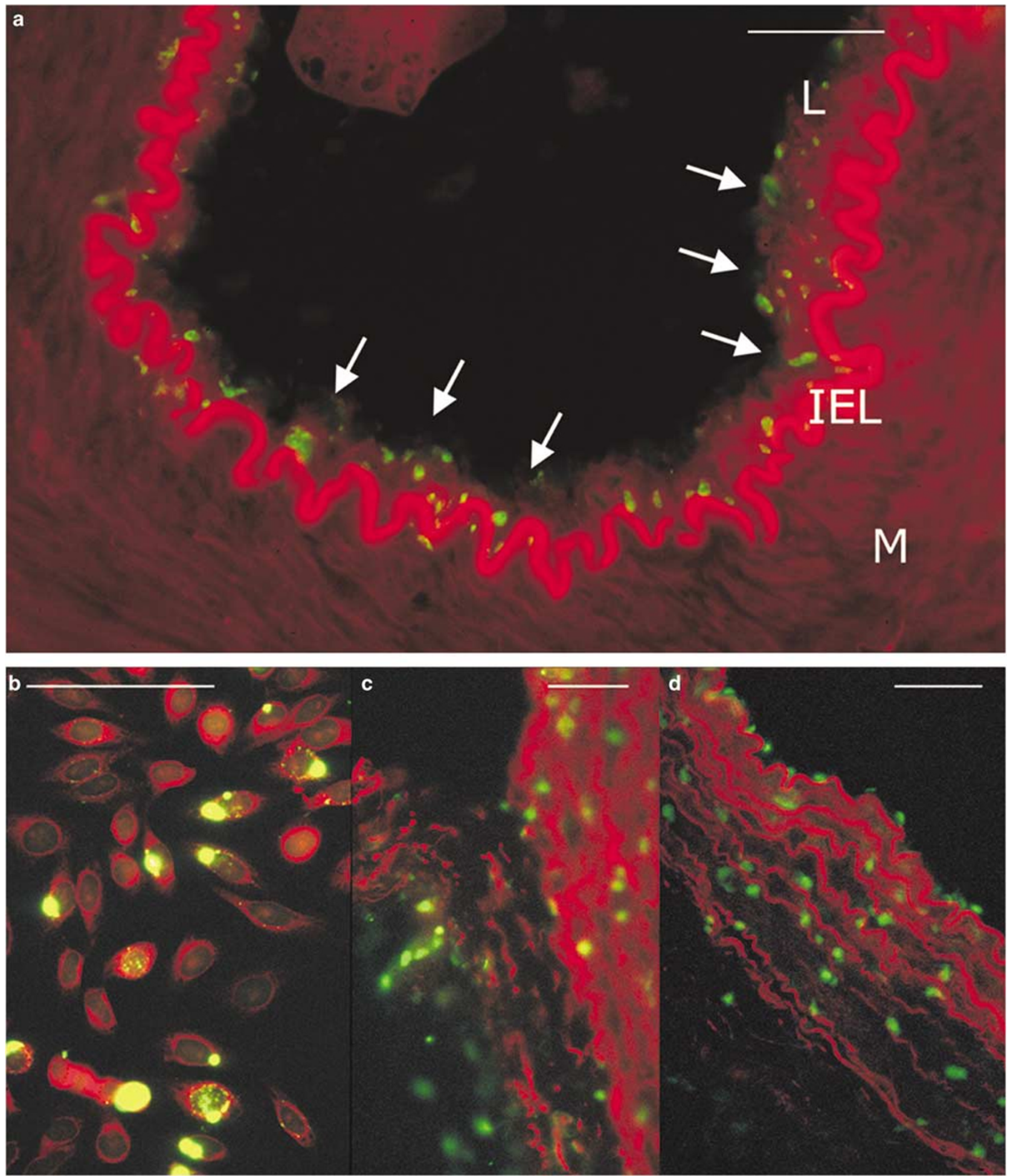

Figure 4 Detection of $C$. pneumoniae (CPN) in chronically infected arteries. (a) Detection of Chlamydophila major outer membrane protein in an atheromatous lesion (L) of a hypercholesterolemic rabbit (arrows). (b) Detection of CPN inclusion bodies in human Hep-2 cells (positive control). CPN was also detected in adventitial tissue (c) and throughout arterial media (d). Immunofluorescence, counterstaining using Evans' blue. IEL: internal elastic membrane, M: media. Bars represent $50 \mu \mathrm{m}$.

the impact of CPN or bacterial components onto a predefined vessel wall segment. Furthermore, sites of injection next to rabbit arteries are not predisposed to shear stress in order to rule out spontaneous lesion formation induced by hypercholesterolemia alone.

The histomorphology of the induced lesions did not differ in all groups treated with CPN or 


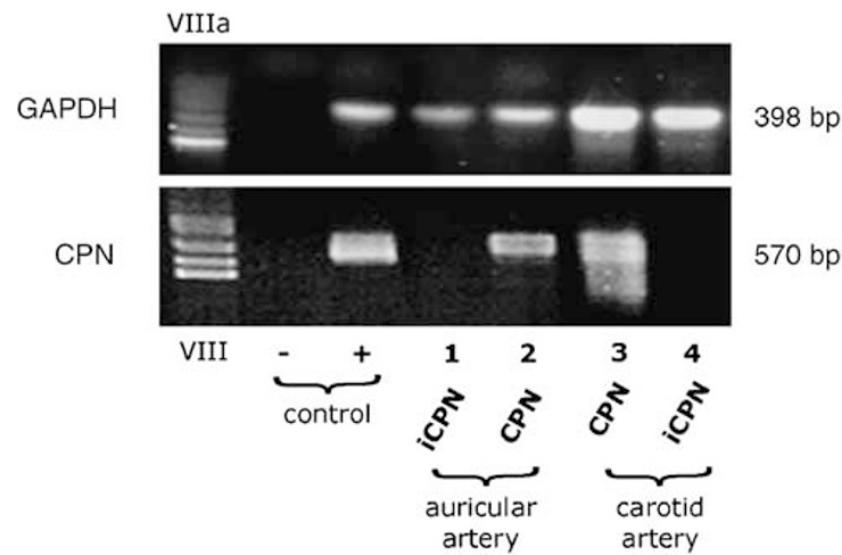

Figure 5 Detection of $C$. pneumoniae (CPN) DNA using nested PCR in chronically infected arteries. Nested PCR demonstrates CPN DNA in vessels following chronic infection with viable organisms (lanes 2, and lane 3), whereas no DNA was detected following application of inactivated CPN (lanes 1, and lane 4). VIII/VIIIa: molecular weight standard.

components, with lesions corresponding to stage II of the Stary classification with predominating macrophages and VSMCs. ${ }^{20}$ More recently, acceleration of complex atherosclerotic lesions was demonstrated in Apo E3-Leiden mice. ${ }^{26}$

The induction of atheromatous lesions induced by CPN AR-39 did not depend on the replication status of bacteria as both viable and heat-inactivated bacteria as well as Gram-negative membrane components such as LPS caused similar effects in vivo. The direct impact of inactivated CPN onto the vessel wall has not yet been compared to viable organisms in vivo. Our findings are in contrast to a recently published study, which demonstrated that heatinactivated CPN organisms are not atherogenic. ${ }^{18}$ In contrast to our model, Sharma et al used a natural infection route via intranasal inoculation of inactivated $\mathrm{CPN}$ in an LDL-/- mice model, suggesting facilitated degradation of inactivated organisms by the natural immune response. However, recent investigations suggested that CPN antigens rather than viable bacteria persist in human atherosclerotic lesions, detecting CPN antigen in the absence of DNA or $16 \mathrm{~S}$ rRNA. ${ }^{27}$ CPN infection promotes a proliferative phenotype in VSMCs via transcription factor Egr-1 activation resulting in accelerated plaque growth. ${ }^{28}$ Local delivery of CPN in LDL receptor -/- mice resulted specifically in pronounced atherosclerotic lesions with a more vulnerable morphology accompanied by a marked upregulation of macrophage chemoattractant protein-1 and intracellular cell adhesion molecule-1, as determined on mRNA and protein level. ${ }^{29,30}$ Moreover, Kalayoglu et al demonstrated that LPS of CPN induces cholesteryl ester accumulation and foam cell formation from macrophages in vitro. This was confirmed by results found in our control group treated with repeated perivascular applications of
E. coli LPS, demonstrating lesion induction, which was prominent, but less intense than following chronic application of CPN (Figure 2). The plaquepromoting effect following local ${ }^{31}$ application was similar to results obtained by Lehr et al using repeated systemic administrations of approximately twice the cumulative dosage of LPS in rabbits, which resulted in increased atherosclerosis as compared with control animals under conditions of hypercholesterolemia. Corresponding with this observation, Gram-negative LPS (from E. coli) induced lipid accumulation and foam cell formation from macrophages in vitro, ${ }^{32}$ a mechanism that may explain increased plaque formation demonstrated in vivo by our group and others. ${ }^{13,33-36}$ Other bacterial components, such as Chlamydia heatshock protein (cHSP) 60, were shown to be associated with the presence of CPN in atheromatous plaques. ${ }^{37} \mathrm{Kol}$ et $a l^{38,39}$ demonstrated that cHSP 60 has been localized in human atheroma and activated human vascular endothelium, smooth muscle cells, and macrophages.

However, plaque progression following chronic CPN infection may result from the vasculotropism and the ability of CPN to persist in VSMCs and atheromatous plaques, ${ }^{4,40,41}$ whereas other bacteria such as $E$. coli or $C$. trachomatis have never been detected in plaque tissues. The vasculotropism of CPN was recently demonstrated in an ex vivo human renal artery model, where LPS from CPN could be detected broadly in arteries following local infection; however, in this study, recultivation of viable organisms failed in all cases. ${ }^{42}$ The atherogenic effect of bacterial products demonstrated in the current investigation did not depend on the systemic inflammatory response, as hypercholesterolemic animals that received repeated applications developed a comparable slight increase of serum CRP.

In contrast to the chronic infection following repeated bacterial application, neither single applications of viable CPN nor inactivated organisms resulted in atheromatous lesion development in hypercholesterolemic rabbits. The lesion promotion effect of the bacterial organisms or components was restricted to conditions of hypercholesterolemia. In normocholesterolemic animals, CPN, inactivated CPN, or LPS were not able to self-induce atheromatous lesions by theirself in the absence of major atherogenic stimuli, suggesting that infectious agents rather act as secondary cofactors. Focal intimal thickening and leukocyte migration after administration of LPS have previously been described in a rat model without cholesterol feeding. ${ }^{43}$ Transient migration of VSMCs into neointima resulting from perivascular inflammation using LPS from $E$. coli in normocholesterolemic rabbits has been described previously by our group. ${ }^{19}$ Further studies on lesion initiation during the early phase of the inflammatory injury are needed as we did not observe significant presence of inflammatory 
cells after 16 weeks of repeated infections using CPN either in its viable or in inactivated form. Moreover, the local inoculation of fractionated bacterial components will allow identification of atherogenic factors.

However, despite evidence from these experimental studies, most clinical trials did not demonstrate beneficial effects of macrolide treatment on clinical outcome or reduction of cardiovascular events in patients suffering from ischemic heart disease. ${ }^{15-17}$ The inability of chlamydial or bacterial components to initiate atheromatous lesions in the absence of major risk factors, such as hypercholesterolemia, as demonstrated in the present study may implicate one reason for failure of antibiotic treatment in coronary disease. Others may be the difficulty in treating chronic chlamydial infections with antimicrobial agents owing to the intracellular nature of the organism and the ability to exist in a persistent, nonreplicative, state within the host. ${ }^{5}$

The current perivascular inflammation model allowed the assessment of the chronic impact of defined dosages of different bacterial pathogens onto predefined sites of the vascular wall in the context of atherogenesis. However, the model is limited by the artificial way of presenting bacterial pathogens to the vascular wall as those bacteria that have been shown to be atherogenic (ie CPN) are usually transmitted via the respiratory tract. The presence of chlamydial major outer membrane protein using immunohistochemistry in all layers of the inoculated arteries implies transport of the organisms into intimal and medial compartments via vasa vasorum or migration of infected VSMCs or macrophages throughout the vessel wall. CPN is able to infect VSMC and to induce aponecrosis of human aortic smooth muscle cells, as recently shown by Dumrese et $a .^{44}$ In addition, further studies may focus on the time course of inflammatory changes and the fate of chlamydial components throughout the vessel wall as we observed a difference in the presence of chlamydial antigen between viable and inactivated bacteria applications.

In summary, chronic perivascular administration of viable CPN next to rabbit arteries, which are not predisposed to shear stress resulted in significant plaque formation, only in the case of hypercholesterolemia. This observation was independent of the replication state of the bacteria as comparable effects were demonstrated following application of inactivated organisms. The plaque-promoting effect was also present by repeated local perivascular administrations of $E$. coli LPS, supporting hypothesis that the response to injury of arteries, following the presence of bacterial products is a nonspecific inflammatory cascade that results in atherogenesis. These data suggest that chronic presence of CPN or CPN components appears as cofactors in atherogenesis requiring other major risk factors such as hypercholesterolemia.

\section{Acknowledgements}

We thank TY Huehns, MRCP, for her help in preparing the manuscript, and $\mathrm{B}$ Leitermeier and I Plonner for technical assistance. We are further indebted to Hans D Theiss, MD, and Anja Schroff, who performed the rabbit CRP ELISA analyses. Finally, we thank Sorin Armeanu, PhD, for his help in designing the study. This work was funded in part by the Research Foundation of the Ludwig Maximilian University, Munich, Germany (MGE, Grant No. 126). This study was presented at the Scientific Sessions 2005 of the American Heart Association, November 13-16, Dallas, TX, USA.

\section{Conflict of interest}

None.

\section{References}

1 Saikku P, Leinonen M, Mattila K, et al. Serological evidence of an association of a novel Chlamydia, TWAR, with chronic coronary heart disease and acute myocardial infarction. Lancet 1988;2:983-986.

2 Kuo CC, Shor A, Campbell LA, et al. Demonstration of Chlamydia pneumoniae in atherosclerotic lesions of coronary arteries. J Infect Dis 1993;167:841-849.

3 Campbell LA, Perez Melgosa M, Hamilton DJ, et al. Detection of Chlamydia pneumoniae by polymerase chain reaction. J Clin Microbiol 1992;30:434-439.

4 Muhlestein JB, Hammond EH, Carlquist JF, et al. Increased incidence of Chlamydia species within the coronary arteries of patients with symptomatic atherosclerotic vs other forms of cardiovascular disease. J Am Coll Cardiol 1996;27:1555-1561.

5 De Kruif MD, van Gorp EC, Keller TT, et al. Chlamydia pneumoniae infections in mouse models: relevance for atherosclerosis research. Cardiovasc Res 2005;65: 317-327.

6 Kaukoranta Tolvanen SS, Laurila AL, Saikku P, et al. Experimental infection of Chlamydia pneumoniae in mice. Microb Pathogen 1993;15:293-302.

7 Kuo CC, Chen HH, Wang SP, et al. Identification of a new group of Chlamydia psittaci strains called TWAR. J Clin Microbiol 1986;24:1034-1037.

8 Yang ZP, Kuo CC, Grayston JT. A mouse model of Chlamydia pneumoniae strain TWAR pneumonitis. Infect Immun 1993;61:2037-2040.

9 Yang ZP, Kuo CC, Grayston JT. Systemic dissemination of Chlamydia pneumoniae following intranasal inoculation in mice. J Infect Dis 1995;171:736-738.

10 Bell TA, Kuo CC, Wang SP, et al. Experimental infection of baboons (Papio cynocephalus anubis) with Chlamydia pneumoniae strain 'TWAR'. J Infect 1989;19:47-49.

11 Moazed TC, Campbell LA, Rosenfeld ME, et al. Chlamydia pneumoniae infection accelerates the progression of atherosclerosis in apolipoprotein E-deficient mice. J Infect Dis 1999;180:238-241.

12 Muhlestein JB, Anderson JL, Hammond EH, et al. Infection with Chlamydia pneumoniae accelerates the development of atherosclerosis and treatment with 
azithromycin prevents it in a rabbit model. Circulation 1998;97:633-636.

$13 \mathrm{Hu} \mathrm{H}$, Pierce GN, Zhong G. The atherogenic effects of chlamydia are dependent on serum cholesterol and specific to Chlamydia pneumoniae. J Clin Invest 1999; 103:747-753.

14 Parchure N, Zouridakis EG, Kaski JC. Effect of azithromycin treatment on endothelial function in patients with coronary artery disease and evidence of Chlamydia pneumoniae infection. Circulation 2002; 105:1298-1303.

15 Anderson JL, Muhlestein JB, Carlquist J, et al. Randomized secondary prevention trial of azithromycin in patients with coronary artery disease and serological evidence for Chlamydia pneumoniae infection: The Azithromycin in Coronary Artery Disease: Elimination of Myocardial Infection with Chlamydia (ACADEMIC) study. Circulation 1999;99:1540-1547.

16 Cercek B, Shah PK, Noc M, et al. Effect of short-term treatment with azithromycin on recurrent ischaemic events in patients with acute coronary syndrome in the Azithromycin in Acute Coronary Syndrome (AZACS) trial: a randomised controlled trial. Lancet 2003;361: 809-813.

17 Anderson JL, Muhlestein JB. Antibiotic trials for coronary heart disease. Tex Heart Inst J 2004;31:33-38.

18 Sharma J, Niu Y, Ge J, et al. Heat-inactivated $C$. pneumoniae organisms are not atherogenic. Mol Cell Biochem 2004;260:147-152.

19 Engelmann MG, Redl CV, Nikol S. Recurrent perivascular inflammation by lipopolysaccharide (endotoxin) results in the formation of atheromatous lesions in vivo. Lab Invest 2004;84:425-432.

20 Stary HC, Chandler AB, Dinsmore RE, et al. A definition of advanced types of atherosclerotic lesions and a histological classification of atherosclerosis. A report from the Committee on Vascular Lesions of the Council on Arteriosclerosis, American Heart Association. Circulation 1995;92:1355-1374.

21 Kuo C, Campbell LA. Is infection with Chlamydia pneumoniae a causative agent in atherosclerosis? Mol Med Today 1998;4:426-430.

22 Capron L. Chlamydia in coronary plaques-hidden culprit or harmless hobo? Nat Med 1996;2:856-857.

23 Moazed TC, Kuo C, Patton DL, et al. Experimental rabbit models of Chlamydia pneumoniae infection. Am J Pathol 1996;148:667-676.

24 Fong IW, Chiu B, Viira E, et al. Rabbit model for Chlamydia pneumoniae infection. J Clin Microbiol 1997;35:48-52.

25 Muhlestein JB. Chlamydia pneumoniae-induced atherosclerosis in a rabbit model. J Infect Dis 2000;181: S505-S507.

26 Ezzahiri R, Nelissen-Vrancken HJ, Kurvers HA, et al. Chlamydophila pneumoniae (Chlamydia pneumoniae) accelerates the formation of complex atherosclerotic lesions in Apo E3-Leiden mice. Cardiovasc Res 2002;56:269-276.

27 Meijer A, Roholl PJ, Gielis-Proper SK, et al. Chlamydia pneumoniae antigens, rather than viable bacteria, persist in atherosclerotic lesions. J Clin Pathol 2000;53: 911-916.

28 Rupp J, Hellwig-Burgel T, Wobbe V, et al. Chlamydia pneumoniae infection promotes a proliferative phenotype in the vasculature through Egr-1 activation in vitro and in vivo. Proc Natl Acad Sci USA 2005;102: 3447-3452.
29 Hauer AD, de Vos P, Peterse N, et al. Delivery of Chlamydia pneumoniae to the vessel wall aggravates atherosclerosis in $\operatorname{LDLr}(-/-)$ mice. Cardiovasc Res 2005;17:17.

30 Kalayoglu MV, Byrne GI. A Chlamydia pneumoniae component that induces macrophage foam cell formation is chlamydial lipopolysaccharide. Infect Immun 1998;66:5067-5072.

31 Lehr HA, Sagban TA, Ihling C, et al. Immunopathogenesis of atherosclerosis: endotoxin accelerates atherosclerosis in rabbits on hypercholesterolemic diet. Circulation 2001;104:914-920.

32 Funk JL, Feingold KR, Moser AH, et al. Lipopolysaccharide stimulation of RAW 264.7 macrophages induces lipid accumulation and foam cell formation. Atherosclerosis 1993;98:67-82.

33 Netea MG, Demacker PN, Kullberg BJ, et al. Increased interleukin-1alpha and interleukin-1beta production by macrophages of low-density lipoprotein receptor knock-out mice stimulated with lipopolysaccharide is CD11c/CD18-receptor mediated. Immunology 1998;95: $466-472$.

34 Leirisalo Repo M, Jaattela M, Gylling $\mathrm{H}$, et al. Phagocyte function in familial hypercholesterolaemia: peripheral blood monocytes exposed to lipopolysaccharide show increased tumour necrosis factor production. Scand J Immunol 1990;32:679-685.

35 Netea MG, Demacker PN, Kullberg BJ, et al. Low-density lipoprotein receptor-deficient mice are protected against lethal endotoxemia and severe Gram-negative infections. J Clin Invest 1996;97: 1366-1372.

36 Rovers C, Netea MG, de Bont N, et al. LPS-induced cytokine production and expression of beta2-integrins and CD14 by peripheral blood mononuclear cells of patients with homozygous familial hypercholesterolemia. Atherosclerosis 1998;141:99-105.

37 Fong IW, Chiu B, Viira E, et al. Chlamydial heat-shock protein-60 antibody and correlation with Chlamydia pneumoniae in atherosclerotic plaques. J Infect Dis 2002;186:1469-1473.

38 Kol A, Sukhova GK, Lichtman AH, et al. Chlamydial heat shock protein 60 localizes in human atheroma and regulates macrophage tumor necrosis factor-alpha and matrix metalloproteinase expression. Circulation 1998;98:300-307.

39 Kol A, Bourcier T, Lichtman AH, et al. Chlamydial and human heat shock protein 60s activate human vascular endothelium, smooth muscle cells, and macrophages. J Clin Invest 1999;103:571-577.

40 Maass M, Krause E, Engel PM, et al. Endovascular presence of Chlamydia pneumoniae in patients with hemodynamically effective carotid artery stenosis. Angiology 1997;48:699-706.

41 Maass M, Bartels C, Engel PM, et al. Endovascular presence of viable Chlamydia pneumoniae is a common phenomenon in coronary artery disease. J Am Coll Cardiol 1998;31:827-832.

42 Poppert S, Schlaupitz K, Marre R, et al. Chlamydia pneumoniae in an ex vivo human artery culture model. Atherosclerosis 2005;29:29.

43 Prescott MF, McBride CK, Court M. Development of intimal lesions after leukocyte migration into the vascular wall. Am J Pathol 1989;135:835-846.

44 Dumrese C, Maurus CF, Gygi D, et al. Chlamydia pneumoniae induces aponecrosis in human aortic smooth muscle cells. BMC Microbiol 2005;5:2. 